\title{
ON THE NUMBER OF GOOD RATIONAL APPROXIMATIONS TO ALGEBRAIC NUMBERS
}

\author{
JULIA MUELLER AND W. M. SCHMIDT
}

(Communicated by William Adams)

\begin{abstract}
We study rational approximations $x / y$ to algebraic and, more generally, to real numbers $\xi$. Given $\delta>0$, and writing $L=\log (1+\delta)_{\text {, the num- }}$ ber of approximations with $|\xi-(x / y)|<y^{-2-\delta}$ is $\leq L^{-1} \log \log H+c_{1}(\delta, r)$ if $\xi$ is algebraic of degree $\leq r$ and of height $H$, and is $\leq L^{-1} \log \log B+c_{2}(\delta)$ if $\xi$ is real and we restrict to approximations with $y \leq B$. It turns out that the dependency on $H$ resp. $B$ in these estimates is the best possible, i.e., that the summands $L^{-1} \log \log H$ resp. $L^{-1} \log \log B$ are optimal.
\end{abstract}

\section{INTRODUCTION}

Our investigation started with the observation that a recent bound of Bombieri and Van der Poorten [1], and also of Luckhardt [4], for the number of exceptional approximations in Roth's Theorem is in some sense the best possible. But it will be convenient to begin our discussion with good approximations to real numbers in general.

Suppose $\delta>0, M>0$. Given a real number $\xi$, a rational number $x / y$ in reduced form and with $y>0$ will be called a $(\delta, M)$-approximation to $\xi$ if

$$
\left|\xi-\frac{x}{y}\right|<\frac{M}{y^{2+\delta}} \text {. }
$$

Throughout, write

$$
L=\log (1+\delta) .
$$

Theorem 1. Suppose $\delta>0, M \geq \frac{1}{2}$, and let $\xi$ be real. Then for any $B>1$, the number of $(\delta, M)$-approximations to $\xi$ with $y \leq B$ is

$$
\leq L^{-1} \log ^{+} \log B+24 M\left(\delta^{-1}+1\right) .
$$

Here and below, $\log ^{+} x=\log x$ when $x>1$, and $\log ^{+} x=0$ when $0<x \leq 1$. The first summand in (3) is the best possible.

Received by the editors June 9, 1988.

1980 Mathematics Subject Classification (1985 Revision). Primary 11J68, $11 \mathrm{~J} 17$.

Key words and phrases. Rational approximation to algebraic numbers.

The first author was supported in part by NSF grant DMS- 8604568 . 
Theorem 2. Suppose $\delta>0, M>0$. Then there is a real transcendental number $\xi$ such that for every $B>\max \left(e,\left(2 M^{-1}+1\right)^{1 / \delta}\right)$, the number of $(\delta, M)$ approximations to $\xi$ with $y \leq B$ is

$$
\geq L^{-1} \log \log B-L^{-1} \log \left(\delta^{-1} \log \left(2 M^{-1}+1\right)\right) .
$$

We now turn to approximations to an algebraic number $\alpha$. Suppose $\alpha$ is algebraic of degree $\leq r$ where $r>1$, and of absolute height $H(\alpha)=H$, as defined, e.g. in [1]. Bombieri and Van der Poorten showed that the number of reduced approximations $x / y$ with positive $y$ and with

$$
\left|\alpha-\frac{x}{y}\right|<\frac{1}{64 h(x / y)^{2+\delta}}
$$

where $h(x / y):=\max (|x|, y)$ and where $0<\delta<\delta_{0}$ with absolute $\delta_{0}$, is

$$
\leq(2 / \delta) \log \log 4 H+3000 \frac{(\log r)^{2}}{\delta^{5}} \log \left(\frac{50 \log r}{\delta^{2}}\right) .
$$

Luckhardt [4] used "Herbrand analysis" to obtain a similar result. Earlier bounds are due to Davenport and Roth [2]. In order to express everything in terms of $(\delta, M)$-approximations, we will easily deduce the following from the Bombieri-Van der Poorten argument.

Theorem 3. With $\alpha$ as above, and with $0<\delta<\delta_{0}, M \geq \frac{1}{2}$, the number of $(\delta, M)$-approximations to $\alpha$ is

$$
<L^{-1} \log ^{+} \log H+2 \cdot 10^{5} M \frac{(\log r)^{2}}{\delta^{5}} \log \left(\frac{200 \log r}{\delta^{2}}\right) .
$$

E.g., for $M=1$, this bound is as good as (4), except for the values of some constants. Again the first summand in the estimate is the best possible.

Theorem 4. Suppose $\delta>0, M>0$.

(i) Let $K$ be an algebraic number field of degree $r$. Then there are infinitely many algebraic numbers $\alpha$ with $K=Q(\alpha)$ such that the number of $(\delta, M)$ approximations to $\alpha$ is

$$
>L^{-1} \log ^{+} \log H(\alpha)-c_{1}(\delta, M, K) .
$$

In the special case when $\delta \leq 1, M=1$ and $K=Q(\sqrt[r]{2})$, we may take

$$
c_{1}(\delta, M, K)=L^{-1} \log (7 / \delta) .
$$

(ii) Given $r>0$, there are infinitely many algebraic numbers $\alpha$ of degree $\leq r$ having at least

$$
L^{-1} \log ^{+} \log H(\alpha)+L^{-1} \log r-c_{2}(\delta, M)
$$

$(\delta, M)$-approximations.

This shows in particular that the second summand in (5) is not superfluous, and that it has to involve $\log r$. 
We will prove Theorems $1-4$ in $\S \S 2$ and 3. In subsequent work [6], the second author will generalize the present results to simultaneous approximations.

\section{Good RATIONAL APPROXIMATIONS TO REAL NUMBERS}

For completeness we begin with a very simple lemma which is probably well known, although not stated explicitly in the literature.

Lemma. Let $\xi$ be real and $\delta>0$. Given $1<A \leq B$, the number of $\left(\delta, \frac{1}{2}\right)$ approximations to $\xi$ with $A \leq y \leq B$ is

$$
\leq 1+L^{-1} \log ^{+}(\log B / \log A) .
$$

Proof. If $x / y$ and $x^{\prime} / y^{\prime}$ are distinct such approximations with $y \leq y^{\prime}$, then

$$
\frac{1}{y y^{\prime}} \leq\left|\xi-\frac{x}{y}\right|+\left|\xi-\frac{x^{\prime}}{y^{\prime}}\right|<\frac{1}{2 y^{2+\delta}}+\frac{1}{2 y^{\prime 2+\delta}} \leq \frac{1}{y^{2+\delta}},
$$

so that we have the "gap principle" $y^{\prime} \geq y^{1+\delta}$. When $x_{0} / y_{0}, \ldots, x_{\nu} / y_{\nu}$ are the $\left(\delta, \frac{1}{2}\right)$-approximations with $A \leq y_{0} \leq y_{1} \leq \cdots \leq y_{\nu} \leq B$, then

$$
A^{(1+\delta)^{\nu}} \leq y_{\nu} \leq B
$$

so that

$$
\nu \leq L^{-1} \log ^{+}(\log B / \log A) .
$$

Proof of Theorem 1. When $x / y$ and $x^{\prime} / y^{\prime}$ are distinct $(\delta, M)$-approximations to $\xi$ with $y \leq y^{\prime}$, then we have the easily derived "gap principle" that $y^{\prime} \geq$ $(2 M)^{-1} y^{1+\delta}$. This is useless unless $y^{\delta}>2 M$. Hence let us call $x / y$ a large approximation if $y>e(2 M)^{1 / \delta}$. If $y_{0} \leq y_{1} \leq \cdots \leq y_{\nu}$ are the denominators belonging to large $(\delta, M)$-approximations, then

$$
\begin{aligned}
\log y_{1} & \geq(1+\delta) \log y_{0}-\log (2 M), \\
\log y_{2} \geq & (1+\delta)^{2} \log y_{0}-((1+\delta)+1) \log (2 M), \\
& \quad \cdots \\
\log y_{\nu} \geq & (1+\delta)^{\nu} \log y_{0}-\left((1+\delta)^{\nu-1}+\cdots+(1+\delta)+1\right) \log (2 M) \\
> & (1+\delta)^{\nu}\left(\log y_{0}-\delta^{-1} \log (2 M)\right) \\
\geq & (1+\delta)^{\nu} .
\end{aligned}
$$

Now when $y_{\nu} \leq B$, we obtain $(1+\delta)^{\nu}<\log B$, so that the number of large approximations is

$$
1+\nu<1+L^{-1} \log ^{+} \log B .
$$

Next, consider the set $S(u)$ of $(\delta, M)$-approximations with $e^{u} \leq y<e^{u+1}$. If $u$ is fixed and if (with a change of notation) $x_{0} / y_{0}, \ldots, x_{\mu} / y_{\mu}$ are the approximations in $S(u)$, ordered such that $x_{0} / y_{0}<\cdots<x_{\mu} / y_{\mu}$, then $x_{i+1} / y_{i+1}$ $x_{i} / y_{i} \geq 1 /\left(y_{i} y_{i+1}\right)>e^{-2 u-2}$, so that $x_{\mu} / y_{\mu}-x_{0} / y_{0}>\mu e^{-2 u-2}$. On the other 
hand, $x_{\mu} / y_{\mu}-x_{0} / y_{0} \leq\left|\xi-\left(x_{\mu} / y_{\mu}\right)\right|+\left|\xi-\left(x_{0} / y_{0}\right)\right|<2 M e^{-u(2+\delta)}$. Thus $S(u)$ has cardinality

$$
|S(u)|=1+\mu<1+2 M e^{2-u \delta} .
$$

The small approximations are contained in the union of $S(0), \ldots, S(k)$ with $k=1+\left[\delta^{-1} \log (2 M)\right]$, where [ ] denotes integer parts. The total number of small approximations is

$$
\begin{aligned}
& \leq \sum_{u=0}^{k}\left(1+2 M e^{2-u \delta}\right) \leq k+1+2 e^{2} M \sum_{u=0}^{\infty} e^{-u \delta}=k+1+2 e^{2} M\left(1-e^{-\delta}\right)^{-1} \\
& \leq 2+\delta^{-1} \log (2 M)+2 e^{2} M\left(\delta^{-1}+1\right)<2+18 M\left(\delta^{-1}+1\right)<22 M\left(\delta^{-1}+1\right) .
\end{aligned}
$$

By (6), the total number of large and small approximations is

$$
<\nu+1+22 M\left(\delta^{-1}+1\right)<L^{-1} \log ^{+} \log B+24 M\left(\delta^{-1}+1\right) \text {. }
$$

Proof of Theorem 2. We define a sequence of positive integers $a_{1}=1, a_{2}, \ldots$ by induction. Set $q_{0}=1$. When $a_{1}, \ldots, a_{n}$ are given, let $p_{n} / q_{n}$ be the continued fraction (see [3], Chapter X)

$$
\frac{p_{n}}{q_{n}}=\left[0, a_{1}, \ldots, a_{n}\right]=\frac{1}{a_{1}+\frac{1}{a_{2}+\ddots \cdot+\frac{1}{a_{n}}} .}
$$

Let $a_{n+1}$ be the least integer with $a_{n+1} q_{n}+q_{n-1} \geq 2 M^{-1} q_{n}^{1+\delta}$. (The factor 2 will only be needed in the proof of Theorem 4 below.) Then $q_{n+1}=a_{n+1} q_{n}+q_{n-1}$ has

$$
2 M^{-1} q_{n}^{1+\delta} \leq q_{n+1}<2 M^{-1} q_{n}^{1+\delta}+q_{n} \leq\left(2 M^{-1}+1\right) q_{n}^{1+\delta} .
$$

Let $\xi$ be the infinite continued fraction $\xi=\left[0, a_{1}, a_{2}, \ldots\right]$. Then

$$
\left|\xi-\frac{p_{n}}{q_{n}}\right|<\frac{1}{q_{n} q_{n+1}} \leq \frac{M}{2 q_{n}^{2+\delta}}<\frac{M}{q_{n}^{2+\delta}} \quad(n=1,2, \ldots),
$$

so that each $p_{n} / q_{n}$ is a $(\delta, M)$-approximation to $\xi$. Writing $Q=\log \left(2 M^{-1}+1\right)$ we have $\log q_{1}=0, \log q_{2} \leq(1+\delta) \log q_{1}+Q=Q, \log q_{3} \leq(1+\delta) \log q_{2}+Q \leq$ $((1+\delta)+1) Q, \ldots$,

$$
\log q_{n} \leq\left((1+\delta)^{n-2}+\cdots+(1+\delta)+1\right) Q<\delta^{-1}(1+\delta)^{n-1} Q .
$$

The number of $(\delta, M)$-approximations with $y \leq B$ is at least $N$, where $N$ is the largest integer satisfying

$$
Q \delta^{-1}(1+\delta)^{N-1} \leq \log B .
$$

We have $Q \delta^{-1}(1+\delta)^{N}>\log B$ and

$$
N>L^{-1} \log ^{+}\left(\delta Q^{-1} \log B\right)=L^{-1} \log \log B-L^{-1} \log \left(\delta^{-1} \log \left(2 M^{-1}+1\right)\right),
$$


since

$$
\log B>\delta^{-1} \log \left(2 M^{-1}+1\right)=\delta^{-1} Q
$$

by our condition on $B$.

In particular, $\xi$ has infinitely many $(\delta, M)$-approximations, so that $\xi$ is transcendental by Roth's Theorem. It would be easy to construct continuummany numbers $\xi$ with the property of Theorem 2 .

\section{GOOD RATIONAL APPROXIMATIONS TO ALGEBRAIC NUMBERS}

Proof of Theorem 3. Initially we will suppose that $|\alpha| \leq \frac{1}{2}$. Let $n$ be minimal with

$$
\delta \sqrt{n} \geq 12 \sqrt{\log r},
$$

and put $\eta=1 /(2 n !)$. A $(\delta, M)$-approximation to $\alpha$ will be called large if

$$
y>(8 M H)^{10 / \delta \eta} .
$$

For such an approximation,

$$
|\alpha-(x / y)|<\frac{M}{y^{2+\delta}}<\frac{1}{64 y^{2+(\delta / 2)}},
$$

since $y^{\delta / 2}>(8 M H)^{5 / \eta}>64 M$. Therefore $|x / y|<|\alpha|+\frac{1}{2} \leq 1$, so that $|x|<y$. Therefore in the notation of [1] with $\beta=x / y$ and $h(\beta):=\max (|x|, y)$, we have

$$
|\alpha-\beta|<\frac{1}{64 h(\beta)^{2+(\delta / 2)}} .
$$

Moreover, since $M \geq 1 / 2$,

$$
4 h(\beta)>y>(4 H)^{10 / \delta \eta},
$$

which is the inequality right at the beginning of the proof of Theorem 2 in [1], but with $\delta / 2$ in place of $\delta$. (But what we call $\delta$ here is $\zeta$ in [1]). Since (10) corresponds to the inequality $\delta \sqrt{n} \geq 6 \sqrt{\log r}$ in [1], it follows from the work of [1] that the number of solutions of (12) and (13) does not exceed the second summand in (4), with $\delta / 2$ in place of $\delta$, i.e.,

$$
3000 \cdot 2^{5} \frac{(\log r)^{2}}{\delta^{5}} \log \left(\frac{200 \log r}{\delta^{2}}\right) \text {. }
$$

There remain the small approximations with $y \leq(8 M H)^{10 / \delta \eta}=B$, say. We have

$$
\begin{aligned}
\log ^{+} \log B & =\log ((10 / \delta \eta) \log (8 M H))=\log (20 n ! / \delta)+\log \log (8 M H) \\
& <\log 20+n \log n+\log \delta^{-1}+\log \log (8 M H)
\end{aligned}
$$

Our choice of $n$ implies that

$$
n \leq 144 \delta^{-2} \log r+1<146 \delta^{-2} \log r,
$$


so that

$$
\log ^{+} \log B<\log \log (8 M H)+150 \delta^{-2}(\log r) \log \left(150 \delta^{-2} \log r\right) .
$$

By Theorem 1, the number of small approximations is

$$
\begin{aligned}
<L^{-1} \log ^{+} \log B+24 M\left(\delta^{-1}+1\right)<L^{-1} \log \log (8 M H)+400 M \delta^{-3}(\log r) \\
\quad \log \left(150 \delta^{-2} \log r\right),
\end{aligned}
$$

provided $0<\delta<\delta_{0}$. Recalling that $M \geq \frac{1}{2}$, and taking the total number of large and small approximations, we get

$$
<L^{-1} \log ^{+} \log H+195,000 M \delta^{-5}(\log r)^{2} \log \left(200 \delta_{-}^{-2} \log r\right) .
$$

All this was when $|\alpha| \leq \frac{1}{2}$. In general, when, say, $\alpha$ has degree $r$ and $K=Q(\alpha)$, the field height of $\alpha$ in $K$ is $H^{r}$, with

$$
H^{r}=\prod_{i=1}^{r} \max \left(1,\left|\alpha^{(i)}\right|\right) \cdot \prod_{v} \max \left(1,|\alpha|_{v}\right),
$$

where $\alpha^{(1)}, \ldots, \alpha^{(r)}$ are the conjugates of $\alpha$, and $v$ runs through the nonArchimedean absolute values. Thus if $m$ is an integer with $|\alpha-m| \leq \frac{1}{2}^{1}$, then $|m| \leq|\alpha|+\frac{1}{2} \leq H^{r}+\frac{1}{2}<2 H^{r}$. The number $\hat{\alpha}=\alpha-m$ has $|\hat{\alpha}| \leq \frac{1}{2}$, and when $\hat{\alpha}^{(i)}$ is a conjugate distinct from $\hat{\alpha}$, then $\left|\hat{\alpha}^{(i)}\right| \leq\left|\alpha^{(i)}\right|+|m| \leq H^{r}+2 H^{r}=3 H^{r}$. Thus when $\widehat{H}$ denotes the absolute height, and therefore $\widehat{H}^{r}$ the field height of $\hat{\alpha}$, we have

$$
\begin{aligned}
\hat{H}^{r} & =\prod_{i=1}^{r} \max \left(1,\left|\hat{\alpha}^{(i)}\right|\right) \prod_{v} \max \left(1,|\hat{\alpha}|_{v}\right) \leq\left(3 H^{r}\right)^{r-1} \prod_{v} \max \left(1,|\alpha|_{v}\right) \\
& \leq\left(3 H^{r}\right)^{r-1} H<3^{r} H^{r^{2}}
\end{aligned}
$$

and $\widehat{H}<3 H^{r}$. Observe that

$$
\log ^{+} \log \hat{H}<\log r+\log \log (3 H)<\log ^{+} \log H+2+\log r .
$$

Since the number of $(\delta, M)$-approximations for $\hat{\alpha}$ is the same as for $\alpha$, it will suffice to use the bound (14) with $H$ replaced by $\widehat{H}$. Theorem 3 follows.

Proof of Theorem 4(i). Let $K$ be a real algebraic number field of degree $r$. Pick a number $\gamma$ with $K=Q(\gamma)$ and with $|\gamma| \leq \frac{1}{2}$. Construct the sequence $\left\{p_{n} / q_{n}\right\}$ as in the proof of Theorem 2. Given $N \geq 1$, let $b_{N}$ be the least integer $\geq M^{-1} q_{N}^{2+\delta}$, and choose the integer $a_{N}$ with

$$
\left|\gamma-\frac{a_{N}}{b_{N}}-\frac{p_{N}}{q_{N}}\right|<\frac{1}{2 b_{N}} \leq \frac{M}{2 q_{N}^{2+\delta}} .
$$

\footnotetext{
${ }^{1}$ We suppose $\alpha$ to be real. In general one may reduce to the case when $|\operatorname{Re} \alpha| \leq \frac{1}{2}$.
} 
Then $\alpha_{N}=\gamma-\left(a_{N} / b_{N}\right)$ generates $K$, and for $1 \leq n \leq N$ we have

$$
\begin{aligned}
\left|\alpha_{N}-\frac{p_{n}}{q_{n}}\right| & \leq\left|\alpha_{N}-\frac{p_{N}}{q_{N}}\right|+\left|\frac{p_{N}}{q_{N}}-\frac{p_{n}}{q_{n}}\right|<\frac{M}{2 q_{N}^{2+\delta}}+\left|\frac{p_{n+1}}{q_{n+1}}-\frac{p_{n}}{q_{n}}\right| \\
& =\frac{M}{2 q_{n}^{2+\delta}}+\frac{1}{q_{n} q_{n+1}} \leq \frac{M}{2 q_{n}^{2+\delta}}+\frac{M}{2 q_{n}^{2+\delta}}=\frac{M}{q_{n}^{2+\delta}}
\end{aligned}
$$

by (7). Thus $\alpha_{N}$ has at least $N(\delta, M)$-approximations.

We next will show that

$$
(2 H(\gamma))^{-1} b_{N} \leq H\left(\alpha_{N}\right) \leq 4 H(\gamma) b_{N} .
$$

For any $\alpha, \beta$ in a number field, we have

$$
\max \left(1,|\alpha-\beta|_{v}\right) \leq \sigma_{v} \max \left(1,|\alpha|_{v}\right) \max \left(1,|\beta|_{v}\right),
$$

where $\sigma_{v}=2$ when $v$ is an Archimedean absolute value, and $\sigma_{v}=1$ when $v$ is a nonArchimedean absolute value. It easily follows that

$$
H(\alpha-\beta) \leq 2 H(\alpha) H(\beta) .
$$

We obtain $H\left(\alpha_{N}\right)=H\left(\gamma-\left(a_{N} / b_{N}\right)\right) \leq 2 H(\gamma) H\left(a_{N} / b_{N}\right)$ and $H\left(a_{N} / b_{N}\right)=$ $H\left(\gamma-\alpha_{N}\right) \leq 2 H(\gamma) H\left(\alpha_{N}\right)$. By (15), $\left|a_{N} / b_{N}\right| \leq|\gamma|+(1 / 2)+\left|p_{N} / q_{N}\right| \leq 2$, so that $b_{N} \leq H\left(a_{N} / b_{N}\right) \leq 2 b_{N}$, and (16) follows.

We obtain $b_{N}<M^{-1} q_{N}^{2+\delta}+1 \leq\left(M^{-1}+1\right) q_{N}^{2+\delta}$ and

$$
H=H\left(\alpha_{N}\right) \leq 4\left(M^{-1}+1\right) H(\gamma) q_{N}^{2+\delta},
$$

so that by (9),

$$
\log H \leq \log \left(4\left(M^{-1}+1\right) H(\gamma)\right)+(2+\delta) \delta^{-1} Q(1+\delta)^{N-1} .
$$

From this one easily gets

$$
N>L^{-1} \log ^{+} \log H-c_{3}(\delta, M, \gamma)
$$

and the first assertion of Theorem $4(\mathrm{i})$.

In the special case when $M=1, \delta \leq 1, K=Q(\sqrt[r]{2})$ and $\gamma=\sqrt[r]{2}-1$, we have $H(\gamma)<\sqrt[r]{2}+1<5 / 2$ and $Q<4 / 3$, so that

$$
\log H<3+(1+(2 / \delta))(4 / 3)(1+\delta)^{N-1}<(7 / \delta)(1+\delta)^{N}
$$

and

$$
N>L^{-1} \log ^{+} \log H-L^{-1} \log (7 / \delta) .
$$

Proof of Theorem 4(ii). Construct $\xi$ as in Theorem 2. By Wirsing's Theorem [7], there are infinitely many algebraic numbers $\alpha$ of degree $\leq r$ with

$$
|\xi-\alpha|<\frac{c_{4}(\xi, r)}{H_{0}(\alpha)^{(r+1) / 2}},
$$

where $H_{0}(\alpha)$ is a certain height function. More precisely, $H_{0}(\alpha)$ is the maximum absolute value of the minimum degree equation satisfied by $\alpha$ over $Q$ 
with coprime integer coefficients. It is known (Mahler [5]) that $(r+1)^{1 / 2} H_{0}(\alpha) \geq$ $H(\alpha)^{r}$. Since our $\xi$ is fixed, we may write $c_{4}(\xi, r)=c_{5}(r)$. Thus

$$
|\xi-\alpha|<\frac{c_{6}(r)}{H(\alpha)^{(r+1) / 2}} .
$$

Pick such an $\alpha$ of large height $H(\alpha)$, and pick $N$ with

$$
\frac{M}{2 q_{N+1}^{2+\delta}} \leq \frac{c_{6}(r)}{H(\alpha)^{(r+1) / 2}}<\frac{M}{2 q_{N}^{2+\delta}} .
$$

For $n \leq N$ we have by $(8)$

$$
\left|\alpha-\frac{p_{n}}{q_{n}}\right| \leq|\alpha-\xi|+\left|\xi-\frac{p_{n}}{q_{n}}\right|<\frac{M}{2 q_{N}^{2+\delta}}+\frac{M}{2 q_{n}^{2+\delta}} \leq \frac{M}{q_{n}^{2+\delta}},
$$

so that $\alpha$ has at least $N(\delta, M)$-approximations. Now

$$
\frac{r+1}{2} \log H(\alpha) \leq(2+\delta) \log q_{N+1}+c_{7}(r, M) \leq(2+\delta) \delta^{-1} Q(1+\delta)^{N}+c_{7}(r, M)
$$

by (18) and (9). Therefore for any $\alpha$ with (17) and $H(\alpha)>c_{8}(r, M)$ we have

$$
\frac{r \delta}{2(2+\delta) Q} \log H(\alpha)<(1+\delta)^{N}
$$

and

$$
N>L^{-1} \log \log H(\alpha)+L^{-1} \log r-L^{-1} \log (((4 / \delta)+2) Q) .
$$

\section{REFERENCES}

1. E. Bombieri and A. J. Van der Poorten, Some quantitative results related to Roth's Theorem, J. Austral. Math. Soc. (Series A) 45 (1988), 233-248.

2. H. Davenport and K. F. Roth, Rational approximations to algebraic numbers, Mathematika 2 (1955), 160-167.

3. G. H. Hardy and E. M. Wright, An introduction to the theory of numbers, 3rd ed., Clarendon Press, Oxford, 1954.

4. H. Luckhardt, Herbrand-Analysen zweier Beweise des Satzes von Roth: polynomiale Anzahlschranken, The Journal of Symbolic Logic, 54 no. 1 (1989), 234-263.

5. K. Mahler, An application of Jensen's formula to polynomials, Mathematika 7 (1960), 98-100.

6. W. M. Schmidt, The number of simultaneous approximations to algebraic numbers, to appear in Proceedings of Conference in honor of L. K. Hau, Bijing, July 1988, Springer-Verlag.

7. E. Wirsing, Approximation mit algebraischen Zahlen beschränkten Grades, J. Reine Angew Math. 206 (1961), 67-77.

Department of Mathematics, Fordham University, Bronx, New York 10458

Department of Mathematics, University of Colorado, Boulder, Colorado 80309 